Bull. Mater. Sci., Vol. 22, No. 1, February 1999, pp. 9-10. (C) Indian Academy of Sciences.

RAPID COMMUNICATION

\title{
On the polar reciprocity model for hot deformation characteristics of materials
}

\author{
S V S NARAYANA MURTY and B NAGESWARA RAO* \\ Materials and Metallurgy Group, *Structural Engineering Group, Vikram Sarabhai Space Centre, \\ Trivandrum 695022, India
}

MS received 4 September 1998

\begin{abstract}
Studies on the models of hot deformation of materials indicate that there is a linear relationship between the dissipation efficiency parameter of the dynamic materials model, and the intrinsic hot workability parameter of the polar reciprocity model, and both models need a stability criterion for delineating the regimes of flow instabilities in the processing maps.
\end{abstract}

Keywords. Hot deformation; workability parameters; processing maps.

\section{Introduction}

Hot workability is concerned with the extent to which a material plastically deforms during shaping at high temperatures without the occurrence of flow localization or fracture. The innate flow behaviour of the material is termed 'intrinsic hot workability' on which studies have become an integral part of material development and its characterization. Kutumbarao and Rajagopalachary (1996) have discussed recent developments in modeling the hot working behaviour of metallic materials. It is noted from their discussion that the predictions of the polar reciprocity model (PRM) and the dynamic materials model (DMM) look very promising for homogeneous materials. In these models, a dimensionless parameter, $\eta$, called the dissipation efficiency parameter used in the DMM (Prasad et al 1984) and the intrinsic hot workability parameter, $\zeta$, used in the PRM (Rajagopalachary and Kutumbarao 1996) are defined in such a way that they can be easily evaluated from the laboratory generated test data. It is stated that in the DMM, the effect of strain history on the hot working schedule is considered to be insignificant. PRM, it was claimed, has been introduced to account for the deformation history dependence of the flow stress while at the same time recognizing the strong rate dependence of the flow stress as the primary flow characteristic of the material. Depending on the nature of the material system and the processing conditions employed, different microscopic phenomena take place in the evolution of structure during hot working. The occurrence of any microscopic phenomenon usually has a bearing on the flow stress

*Author for correspondence $(\sigma)$, which is a function of the strain $(\varepsilon)$, strain rate $(\dot{\varepsilon})$ and temperature $(T)$.

When the material exhibits ideal plastic flow, it was claimed (Kutumbarao and Rajagopalachary 1996) that $\eta$ and $\zeta$ sum to unity and the predictions of PRM are identical to those of DMM. As per the expectations of Kutumbarao and Rajagopalachary (1996), $\zeta \rightarrow 1$, is a necessary condition for the identification of flow instability and the superposition of a separate stability condition as followed in DMM (Prasad 1990), on the $\zeta$ maps is unnecessary. Careful examination of these models indicate that whether the material exhibits ideal plastic flow or not, a linear relationship exists between $\eta$ and $\zeta$, and a separate stability condition is essential for delineating the regimes of flow instabilities in the processing maps.

The purpose of this study is to examine the relationship between $\eta$ and $\zeta$ and suggest a simple procedure to evaluate these parameters.

\section{The dynamic material model}

Prasad et al (1984) proposed the DMM approach which is based on thermodynamic considerations and does not rely on the microstructural behavioural description of the material. According to this approach, the instantaneous power $P$ is dissipated by partitioning into the dissipator content, $G$ and the dissipator cocontent, $J$ as

$$
P=G+J,
$$

or

$$
\sigma \dot{\varepsilon}=\int_{0}^{\dot{\varepsilon}} \sigma \mathrm{d} \dot{\varepsilon}+\int_{0}^{\sigma} \dot{\varepsilon} \mathrm{d} \sigma .
$$


The efficiency of power dissipation, $\eta$ is given by

$$
\eta=\frac{J}{J_{\max }}
$$

where

$$
J_{\max }=1 / 2 \sigma \dot{\varepsilon},
$$

is used to normalize $J$.

\section{The polar reciprocity model}

In PRM (Rajagopalachary and Kutumbarao 1996), the two integrals in (2) are referred to as hardening power, $\dot{W}_{\mathrm{H}}$ and dissipative power, $W_{\mathrm{D}}$, respectively. In this model an intrinsic hot workability parameter, $\zeta$ is defined as

$$
\zeta=\left\{\frac{\dot{W}_{\mathrm{H}}}{\dot{W}_{\mathrm{H}_{\min }}}-1\right\},
$$

where

$$
\dot{W}_{\mathrm{H}_{\min }}=\sigma \dot{\varepsilon} / 2 \text {. }
$$

\section{Linear relationship between $\eta$ and $\zeta$}

From (1) to (6), one can write,

$$
\begin{aligned}
& \eta=\frac{J}{J_{\max }}=\frac{(P-G)}{J_{\max }}=\left(2-\frac{2 G}{\sigma \dot{\varepsilon}}\right), \\
& \zeta=\left(\frac{\dot{W}_{\mathrm{H}}}{\dot{W}_{\mathrm{H}_{\min }}-1}\right)=\left(\frac{2 G}{\sigma \dot{\varepsilon}}-1\right) .
\end{aligned}
$$

Adding (7) and (8) one can get

$$
\eta+\zeta=1
$$

\section{Validation of the linear relationship}

The constitutive equation used by Rajagopalachary and Kutumbarao (1996) in the PRM is

$$
\sigma=\sigma_{\mathrm{b}}+k^{\prime}(\dot{\varepsilon})^{m^{\prime}}
$$

where $\sigma_{\mathrm{b}}$ and $k^{\prime}$ are different functions of strain history. They obtain the intrinsic hot workability parameter

$$
\zeta=1-\left(\frac{\sigma-\sigma_{\mathrm{b}}}{\sigma}\right)\left(\frac{2 m^{\prime}}{m^{\prime}+1}\right),
$$

which can also be obtained using (10) in (8) by evaluating G.

Using the constitutive equation (10) in (7), the efficiency of power dissipation is obtained as

$$
\eta=\left(\frac{\sigma-\sigma_{\mathrm{b}}}{\sigma}\right)\left(\frac{2 m^{\prime}}{m^{\prime}+1}\right)
$$

Equations (11) and (12) satisfy exactly the relationship in (9).

\section{Conclusions}

The efficiency of power dissipation, $\eta$ or the intrinsic hot workability parameter, $\zeta$ depends mainly on the constitutive equation which relates the flow stress $(\sigma)$ to the strain $(\varepsilon)$, strain rate $(\dot{\varepsilon})$ and temperature $(T)$. The constitutive equations are always derived from experimental results and there is no guarantee that the constitutive equation (10) is valid for all materials. It is better to evaluate $G$ by numerical quadrature from the test data through a cubic spline fit and then determine either $\eta$ or $\zeta$ for developing processing maps. As per the expectation of Kutumbarao and Rajagopalachary (1996), the condition $\zeta \rightarrow 1$, for flow instabilities, (9) gives $\eta \rightarrow 0$. This implies that $J=0$ and $P=G$. In this case all of the power would be dissipated by heat and this could lead to plastic instability by a continuum process such as adiabatic shearing. The qualitative condition, $\zeta \rightarrow 1$ proposed in PRM is inadequate for delineating the regimes of instabilities in the processing maps. The above discussions lead to a qualitative check for the stable material flow by $\eta>0$ or $\zeta<1$. Since there is no unique stability criterion which is applicable for all materials, one has to establish a suitable stability criterion for the intended materials by validating the methodology through microstructural observations on the tested specimens.

\section{References}

Kutumbarao V V and Rajagopalachary T 1996 Bull. Mater. Sci. 19677

Prasad Y V R K, Gegel H L, Doraivelu S M, Malas J C, Morgan J T, Lark K A and Barker D R 1984 Metall. Trans. A15 1883

Prasad Y V R K 1990 Indian J. Technol. 28435

Rajagopalachary T and Kutumbarao V V 1996 Scr. Mater. 35 305 